\title{
Covid-19 em pacientes com câncer: um enfoque clinico-epidemiológico
}

Covid-19 in cancer patients: a clinical-epidemiological approach

Covid-19 en pacientes con cáncer: un enfoque clínico-epidemiológico

Paulo Sérgio da Paz Silva Filho ORCID: https://orcid.org/0000-0003-4104-6550 Centro Universitário Maurício de Nassau, Brasil E-mail: pauloosergio1@ outlook.com

Maria Vitalina Alves de Sousa ORCID: https://orcid.org/0000-0003-4448-2489 Centro Universitário INTA, Brasil

E-mail: enf.vitalinaalves@gmail.com Milena Matos da Cruz ORCID: https://orcid.org/0000-0002-9875-8906 ITPAC, Brasil

E-mail: milenamc_5@hotmail.com

Vicente de Paula Freire da Silva Junior ORCID: https://orcid.org/0000-0002-2021-3818 ITPAC, Brasil

E-mail: vicentejunior96@hotmail.com

Karina Raminho Melo

ORCID: https://orcid.org/0000-0001-7094-2042

Universidade Federal do Pará, Brasil

E-mail: Karinaramonho95@gmail.com

Regina Célia da Silva

ORCID: https://orcid.org/0000-0002-6636-5999

Universidade Federal do Piauí, Brasil

E-mail: regina.dasilva@hotmail.com

José Nilton de Araújo Gonçalves

ORCID: https://orcid.org/0000-0002-1578-3656

Universidade Federal de Mato Grosso, Brasil

E-mail: josenilton.ifpi@hotmail.com

José Guilherme Férrer Pompeu

ORCID: https://orcid.org/0000-0001-9038-0833

Universidade Federal do Piaú, Brasil

E-mail: gpompeu8@gmail.com

Levi David de Sousa Moura

ORCID: https://orcid.org/0000-0002-4199-4940

Universidade Federal do Piauí, Brasil

E-mail: levi01-12@hotmail.com

Danilo Martins de Alencar

ORCID: https://orcid.org/0000-0003-1073-3243

Instituto de Ensino Superior do Vale do Parnaíba, Brasil

E-mail: martins-alencar@hotmail.com

Fabiane Jesus da Silva

ORCID: https://orcid.org/0000-0002-8604-3807

Centro Universitário Santo Agostinho, Brasil

E-mail: fabhinie@hotmail.com

Maria Eliane Martins Oliveira da Rocha

ORCID: https://orcid.org/0000-0002-7743-6107

Instituto Fernandes Filgueira, Brasil

Fundação Oswaldo Cruz, Brasil

E-mail: memor10@hotmail.com

Kacilia Bastos de Castro Rodrigues

ORCID: https://orcid.org/0000-0003-0068-2390

Faculdade Aliança, Brasil

E-mail: kaciliacastro@hotmail.com

Luciano Paes Landim Ribeiro

ORCID: https://orcid.org/0000-0002-5495-7938

Centro Universitário Uninovafapi, Brasil

E-mail: lucym_srn@hotmail.com

Joice Mara Ferreira Dos Santos ORCID: https://orcid.org/0000-0002-3231-750X

Associação de Ensino Superior do Piauí, Brasil E-mail: joicinha2.2kinha@gmail.com 
Rayslane Fernandes da Costa ORCID: https://orcid.org/0000-0003-0782-6297 Centro Universitário Maurício de Nassau, Brasil E-mail: rayslane02@gmail.com

Greice Miranda Duarte ORCID: https://orcid.org/0000-0002-6667-7247 Universidade Federal do Piaú, Brasil E-mail: greicemd2015@gmail.com

Thalia Aguiar de Souza

ORCID: https://orcid.org/0000-0003-3792-1252 Centro Universitário INTA, Brasil

E-mail: Thaliaaguiar32@gmail.com

\begin{abstract}
Resumo
O presente estudo tem como objetivo levantar um enfoque Clinico-epidemiológico sobre a Covid-19 em Pacientes com câncer. O presente estudo utilizou como abordagem metodológica de pesquisa a revisão integrativa. Elegeu-se como questão norteadora: "Quais os principais sinais clínicos em pacientes oncológicos com Covid-19?" e "Qual a epidemiologia do câncer juntamente com a covid-19?". Para a realização desse estudo, foram consultadas as bases de dados: MEDLINE, Scholar Google, LILACS, SCIELO e Repositórios. Utilizou-se os Descritores em Ciências da Saúde (DeCS) de forma associada: COVID-19, Câncer, Epidemiologia, Clinica e Coronavírus. Inicialmente, 165 artigos foram encontrados. Destes, 82 foram selecionados para a leitura completa. Os casos discordantes foram deliberados por consenso, resultando na seleção final de 12 artigos para a leitura na íntegra e elaboração deste estudo, onde possuíam informações clínicas e epidemiológicas de pacientes com câncer que foram infectados pelo novo coronavírus. As pessoas com idade avançada e aquelas com doenças crônicas, como diabetes, doenças cardiovasculares e respiratórias, e com imunidade baixa, possuem um risco maior de terem complicações graves se forem contaminadas pelo coronavírus. Da mesma forma, pessoas com câncer que estejam em tratamentos de quimioterapia, radioterapia, que tenham feito cirurgia há menos de um mês ou que façam uso de medicamentos imunossupressores fazem parte do grupo de risco, tendo em vista que pacientes oncológicos são efetivamente mais vulneráveis a desenvolver a COVID-19 em sua forma mais severa.
\end{abstract}

Palavras-chave: COVID-19; Câncer; Epidemiologia; Clinica; Coronavírus.

\begin{abstract}
This study aims to raise a Clinical-epidemiological focus on Covid-19 in cancer patients. The present study used the integrative review as a methodological research approach. The following guiding question was chosen: "What are the main clinical signs in cancer patients with Covid-19?" and "What is the epidemiology of cancer together with covid19?". To carry out this study, the following databases were consulted: MEDLINE, Scholar Google, LILACS, SCIELO and Repositories. The Health Sciences Descriptors (DeCS) were used in an associated way: COVID-19, Cancer, Epidemiology, Clinical and Coronavirus. Initially, 165 articles were found. Of these, 82 were selected for full reading. Discordant cases were deliberated by consensus, resulting in the final selection of 12 articles for full reading and preparation of this study, which contained clinical and epidemiological information on cancer patients who were infected by the new coronavirus. People with advanced age and those with chronic illnesses such as diabetes, cardiovascular and respiratory diseases, and with low immunity, are at greater risk of having serious complications if they are contaminated by the coronavirus. Likewise, people with cancer who are undergoing chemotherapy treatments, radiotherapy, who have undergone surgery for less than a month or who use immunosuppressive drugs are part of the risk group, considering that cancer patients are effectively more vulnerable to develop COVID-19 at its most severe.
\end{abstract}

Keywords: COVID-19; Cancer; Epidemiology; Clinical; Coronavirus.

\title{
Resumen
}

Este estudio tiene como objetivo generar un enfoque clínico-epidemiológico sobre Covid-19 en pacientes con cáncer. El presente estudio utilizó la revisión integradora como enfoque de investigación metodológica. Se eligió la siguiente pregunta orientadora: "¿Cuáles son los principales signos clínicos en pacientes con cáncer con Covid-19?" y “¿Cuál es la epidemiología del cáncer junto con el covid-19?". Para la realización de este estudio se consultaron las siguientes bases de datos: MEDLINE, Scholar Google, LILACS, SCIELO y Repositories. Los Descriptores de Ciencias de la Salud (DeCS) se utilizaron de forma asociada: COVID-19, Cáncer, Epidemiología, Clínica y Coronavirus. Inicialmente se encontraron 165 artículos. De estos, 82 fueron seleccionados para lectura completa. Los casos discordantes fueron deliberados por consenso, resultando en la selección final de 12 artículos para lectura completa y elaboración de este estudio, que contenía información clínica y epidemiológica sobre pacientes oncológicos infectados por el nuevo coronavirus. Las personas con edad avanzada y aquellas con enfermedades crónicas como diabetes, enfermedades cardiovasculares y respiratorias, y con baja inmunidad, tienen mayor riesgo de tener complicaciones graves si se contaminan por el coronavirus. Asimismo, las personas con cáncer que se someten a tratamientos de quimioterapia, radioterapia, que se han sometido a cirugía durante menos de un mes o que usan fármacos 
inmunosupresores forman parte del grupo de riesgo, considerando que los pacientes con cáncer son efectivamente más vulnerables a desarrollar COVID-19 en su máxima expresión. grave.

Palabras Clave: COVID-19; Cáncer; Epidemiología; Clínica; Coronavirus.

\section{Introdução}

Em dezembro de 2019, uma doença respiratória grave, de etiologia desconhecida, foi descoberta na cidade de Wuhan, China. Alguns dias depois, foi descrita como uma doença infecciosa causada pelo novo coronavírus (Severe Acute Respiratory Syndrome Coronavirus-2 ou Sars-Cov-2) e denominada Coronavirus disease 2019 (covid-19). Por conta da sua rápida disseminação e ao aumento do número de casos da doença em diferentes partes do mundo, no dia 11 de março de 2020 , a Organização Mundial de Saúde (OMS) determinou a doença como uma pandemia (Silva et al., 2021).

O período de incubação da infecção gera em torno de 5 a 6 dias, com intervalo que pode durar até 14 dias. O período médio de transmissibilidade dos pacientes infectados pode durar até 7 dias após o início dos sintomas, porém já sabe-se que pacientes que não apresentam sintomas podem transmitir o vírus. A transmissão ocorre através de gotículas de saliva infectadas, inalação ou contato direto com elas. Além disso, evidências crescentes mostram para a rota de transmissão fecal-oral (Zuil et al., 2021).

Os casos podem variar desde formas assintomáticas até o desenvolvimento de pneumonia grave. Porém, entre os sintomas mais frequentes encontram-se a tosse, pirexia, rinorreia, A transmissão viral, esta pode ocorrer por contato, fômites e por gotículas. Por conta disso, técnicas de higiene das mãos e etiquetas respiratórias, cuidados ao tossir e/ou espirrar, ajudam a prevenir sua disseminação (Soares et al., 2021).

O quadro de imunossupressão de alguns pacientes com câncer eleva o risco de infecção por COVID-19 em comparação com a população em geral. A imunossupressão também pode apresentar manifestações graves nesses pacientes com câncer, resultando assim, em atraso no tratamento e hospitalizações desnecessárias que podem afetar negativamente o prognóstico da doença (Al-Quteimat; Amer, 2020).

Pacientes acometido pelo câncer têm um risco aumentado de infecções graves, com um aumento com cerca de 3,5 vezes no risco de precisar de ventilação mecânica ou admissão na UTI ou evoluir para o óbito quando comparado com pacientes sem câncer. Um dos fatores é causado pela malignidade e tratamentos anticâncer, como quimioterapia ou cirurgia. Pois esses pacientes que receberam quimioterapia ou foram submetidos a cirurgia nos 30 dias antes de apresentarem COVID-19 apresentaram maior risco de eventos graves quando comparado com os pacientes que não passaram por esses procedimentos (Liang et al., 2020).

Um dos desafios mais frequente nesse meio é a definição de caso suspeito. Pois sabe-se que aa dispneia é um dos principais sintomas no câncer avançado e, também, pode ser encontrado em casos de atendimento e internação em pacientes com COVID-19. Quando definido como suspeito por infecção por COVID-19, o paciente deve ficar internado isolado, caso ocorra o óbito, há restrições para o reconhecimento do corpo, velório e sepultamento (Sampaio; Dias; Freitas, 2020).

O presente estudo tem como objetivo levantar um enfoque Clinico-epidemiológico sobre a Covid-19 em Pacientes com câncer.

\section{Metodologia}

O presente estudo utilizou como abordagem metodológica de pesquisa a revisão integrativa que tem como principal finalidade criar uma fonte de conhecimento atual sobre o problema selecionado e definir se o conhecimento é apropriado para ser transferido para a prática, incluindo artigos científicos que detalhassem as características clínicas e epidemiológicas dos pacientes oncológicos com Covid-19. Sendo assim, para construir uma revisão integrativa deve-se seguir um rigoroso percurso 
metodológico, o que viabilizará ao leitor verificar as particularidades dos estudos investigados e fornecer contribuições para a evolução e aprimoramento da prática baseada em evidências científicas (Pompeo; Rossi; Galvão, 2009).

Elegeu-se como questão norteadora: “Quais os principais sinais clínicos em pacientes oncológicos com Covid-19?” e "Qual a epidemiologia do câncer juntamente com a covid-19?"

$\mathrm{Na}$ construção da revisão, foram seguidas as seguintes etapas: levantamento da questão de pesquisa; busca na literatura; categorização dos estudos; avaliação dos estudos incluídos na revisão; interpretação dos resultados e apresentação da síntese de conhecimento (Mendes; Silveira; Galvão, 2008).

Para a realização desse estudo, foram consultadas as bases de dados: Medical Literature Analysis and Retrieval System Online (MEDLINE), Scholar Google, Literatura Latino-americana e do Caribe em Ciências da Saúde (LILACS), Scientific Eletronic Library Online (SCIELO) e Repositórios. Utilizou-se os Descritores em Ciências da Saúde (DeCS) de forma associada: COVID-19, Câncer, Epidemiologia, Clinica e Coronavírus.

Em relação aos critérios de inclusão, foram selecionados os artigos que apresentavam dados originais e secundários sobre a associação entre câncer e covid-19. Sendo artigos disponibilizados na íntegra, disponíveis nos idiomas português e inglês e publicados entre os anos de 2019 e 2021. Os critérios de exclusão foram: artigos incompletos, cartas ao editor, debates, resenhas, resumos ou artigos publicados em anais de eventos, indisponíveis na íntegra e duplicados.

A análise e a interpretação dos dados foram feitas por meio da descrição dos achados. Os dados foram organizados em uma tabela Excel®, que compreendeu as seguintes colunas de sintetização: título do estudo, base de dados, periódico, ano de publicação, contexto/local de estudo, desenho metodológico, resultados e conclusões.

Embora esse tipo de trabalho dispense o processo de submissão a um comitê de ética, foram respeitados os princípios éticos da pesquisa ao citar as obras e assim resguardar os direitos autorais dos estudos citados.

\section{Resultados e Discussão}

Inicialmente, 165 artigos foram encontrados. Destes, 82 foram selecionados para a leitura completa. Os casos discordantes foram deliberados por consenso, resultando na seleção final de 12 artigos para a leitura na íntegra e elaboração deste estudo, onde possuíam informações clínicas e epidemiológicas de pacientes com câncer que foram infectados pelo novo coronavírus (Figura 1 e Quadro 1). 
Figura 1. Fluxograma de seleção dos artigos.

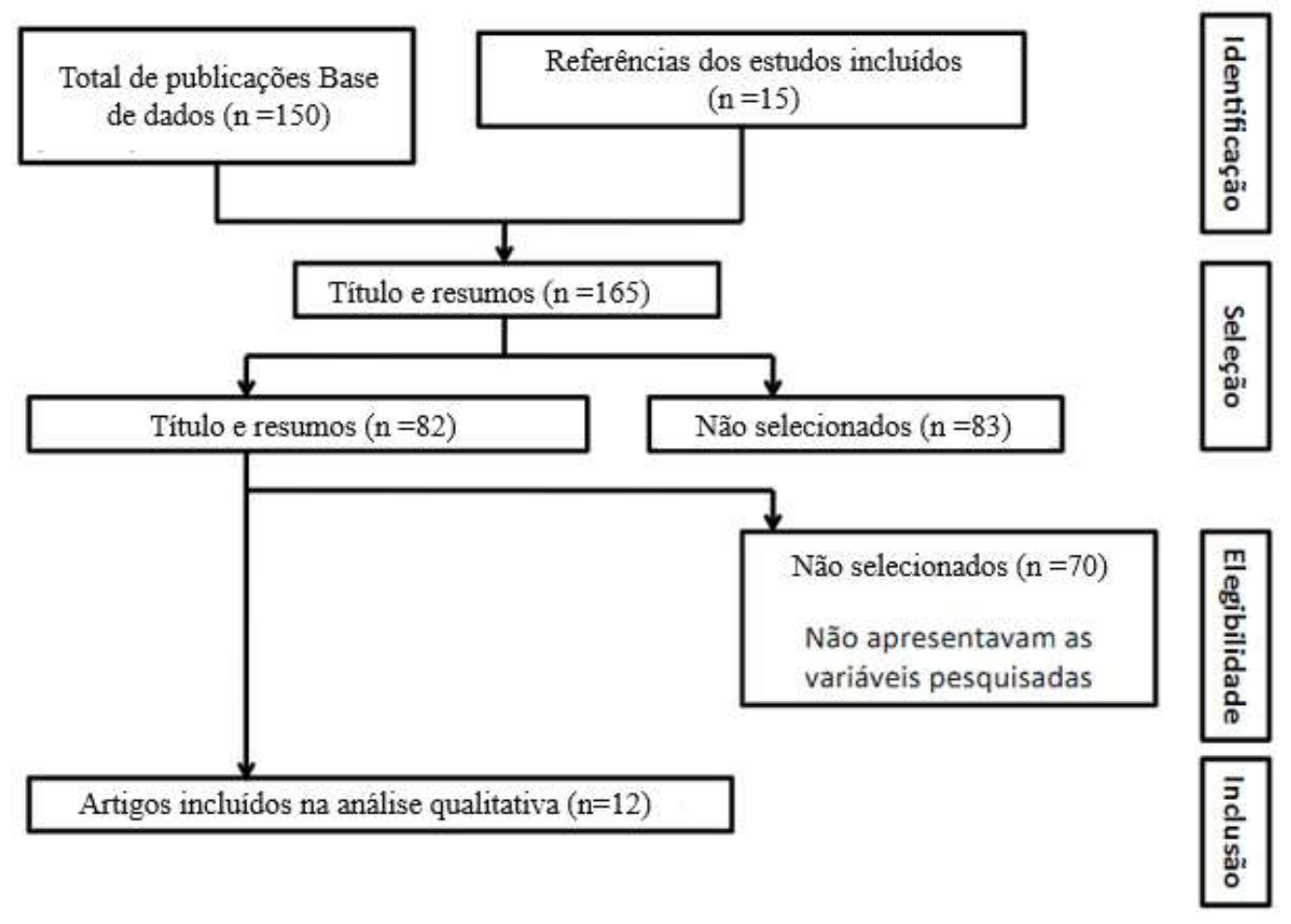

Fonte: Autores (2021).

Quadro 1. Caracterização geral dos artigos incluídos no estudo.

\section{TITULO}

Recomendações para detecção
precoce de câncer durante a
pandemia de covid-19 em
2021.

AUTORES / ANO

(Santos; Corrêa, 2020).

\section{Cancer disease progression} and death during the COVID19 pandemic: a multidisciplinary analysis for the Peruvian setting.
(Astigueta-Pérez et al., 2020).

OBJETIVO

Em geral, o objetivo deve ser agilizar o processo decisório, bem como otimizar e oportunizar ao máximo as ações mais efetivas de deteç̧ão precoce de câncer considerando o monitoramento constante da situação da pandemia no nível local.

Realizar uma análise multidisciplinar usando o diagrama de Ishikawa para identificar os prováveis fatores

que contribuem para a progressão do câncer e óbitos no Peru.

\section{Prevalence of underlying diseases in hospitalized patients with COVID-19: a systematic review and meta- analysis.}

(Emami et al., 2020).

Verificar a prevalência de doenças subjacentes em pacientes hospitalizados com COVID-19

Compilar as informações de

Covid-19 e câncer: atualização de aspectos epidemiológicos.
(Ferreira; Lima; Oliveira, 2020), publicações sobre a infecção por Sars- -CoV-2 em pacientes oncológicos com o

\section{CONCLUSÃO}

Para o bom funcionamento da regulação, é preciso haver clareza dos serviços de referência capazes de levar a termo a investigação diagnóstica de forma conclusiva. Recomenda-se ainda que haja coordenação desse processo de investigação diagnóstica de forma a identificar indivíduos com falhas no processo de seguimento.

Em mais de 120 dias após a declaração do estado de emergência sanitária por COVID19 no Peru, e a partir da análise realizada, há evidências indiretas que sugerem um potencial aumento da morbidade, progressão da doença e mortalidade relacionada ao câncer.

De acordo com os achados do presente estudo, hipertensão, doenças cardiovasculares, diabetes mellitus, tabagismo, doença pulmonar obstrutiva crônica (DPOC), malignidade e doença renal crônica estavam entre as doenças de base mais prevalentes entre pacientes com COVID-19 hospitalizados, respectivamente.

Uma vez que a idade avançada e as comorbidades foram apontadas como fatores de risco, e o câncer pode ser considerado uma doença relacionada ao envelhecimento, 
intuito de alertar e direcionar a assistência prestada por familiares, cuidadores e profissionais de saúde.

\section{Pacientes com câncer na infecção por SARS-CoV-2: uma análise nacional na China.}

(Liang et al., 2020).

Realizar uma análise em pacientes com câncer na infecção por SARS-CoV-2

\section{Desafios na prestação de cuidados com o câncer em meio ao surto da doença coronavírus 19 (COVID-19): precauções específicas para pacientes com câncer e prestadores de cuidados com o câncer para prevenir a disseminação.} oncológicos: uma Revisão do perfil Clínico-Epidemiológico.
Covid-19 em Pacientes

(Shankar et al., 2020).

Informar os desafios no atendimento ao câncer em meio à doença por coronavírus - 19 (COVID-19)
(Alcântara et al., 2020).
Sintetizar a literatura no que se refere ao perfil clínicoepidemiológico de pacientes oncológicos com Covid-19.

\section{Clinical characteristics of COVID-19-infected cancer} patients: a retrospective case study in three hospitals within Wuhan, China
(Zhang et al., 2020).

Descrever as características clínicas de pacientes com câncer infectados por COVID19 pacientes oncológicos são efetivamente mais vulneráveis a desenvolver a Covid-19 em sua foram mais severa.

Os pacientes com câncer tiveram resultados piores do COVID-19, fornecendo um lembrete oportuno aos médicos de que atenção mais intensiva deve ser dada aos pacientes com câncer, em caso de rápida deterioração.

Os países devem isolar, testar, tratar e rastrear para controlar a pandemia de coronavírus. Há uma escassez de informações sobre novas infecções por coronavírus e seu impacto em pacientes com câncer e prestadores de cuidados com o câncer. Até o momento, não há diretrizes científicas sobre o manejo de pacientes com câncer em um contexto de surto de coronavírus.

Muitos esforços estão sendo empreendidos para melhor entender as particularidades dos pacientes com câncer infectados pelo novo coronavírus.

Pacientes com câncer apresentam condições de deterioração e resultados ruins com a infecção por COVID-19. Recomenda-se que pacientes com câncer recebendo tratamentos antitumorais façam triagem vigorosa para infecção por COVID-19 e evitem tratamentos que causem imunossupressão ou tenham suas dosagens reduzidas em caso de coinfecção por COVID-19.

No geral, a evidência atual permanece insuficiente para explicar uma associação conclusiva entre câncer e COVID-19.

Os resultados deste estudo mostram que houve um aumento da mortalidade por câncer de mama no período da pandemia do COVID-19 no Brasil, e que este aumento pode estar atribuído em alguns casos à infecção dessas mulheres pela doença COVID-19, no qual pode-se obter um desfecho desfavorável devido a imunossupressão dessas mulheres Embora os sites participantes tenham sido fortemente encorajados a identificar de forma abrangente os pacientes com câncer
Caracterizar os resultados de uma coorte de pacientes com câncer e COVID-19 e

Clinical impact of COVID-19 on patient with cancer (CCC19): a cohort study
(Kuderer et al., 2020). identificar fatores prognósticos potenciais para mortalidade $\mathrm{e}$ doença grave.
The impact of the COVID19 pandemic on cancer deaths due to delays in
(Maringe,et al., 2020).
Estimar o impacto dos atrasos no diagnóstico sobre os resultados de sobrevida do simultâneo e diagnósticos COVID-19, o viés de seleção é provável, visto que os pacientes que são testados são geralmente sintomáticos e os limiares para teste são mais baixos em ambientes hospitalares. As práticas comunitárias estão um tanto subrepresentadas nesta amostra inicial; portanto, esta coorte pode refletir casos mais graves de COVID-19.

Aumentos substanciais no número de mortes por câncer evitáveis na Inglaterra são esperados como resultado de atrasos no 


\begin{tabular}{c|c|c}
$\begin{array}{c}\text { diagnosis in England, UK: a } \\
\text { national, population-based, } \\
\text { modelling study. }\end{array}$ & $\begin{array}{c}\text { câncer em quatro tipos } \\
\text { principais de tumor. }\end{array}$ & $\begin{array}{c}\text { diagnóstico devido à pandemia de COVID-19 } \\
\text { no Reino Unido. São necessárias intervenções } \\
\text { políticas urgentes, particularmente a } \\
\text { necessidade de gerenciar o acúmulo nos } \\
\text { serviços de diagnóstico de rotina para mitigar } \\
\text { o impacto esperado da pandemia de COVID- } \\
19 \text { em pacientes com câncer. }\end{array}$ \\
\hline
\end{tabular}

Fonte: Autores (2021).

Com a pandemia da doença ocasionada pela COVID-19, os atendimentos eletivos, incluindo o rastreamento de câncer, foram suspensas em quase todo mundo por conta da priorização das urgências e da redução do risco de disseminação do novo coronavírus (SARS-CoV-2) nos serviços de saúde (Santos; Corrêa, 2020).

O uso de ações para controlar e gerenciar o câncer em países em desenvolvimento é crítica, mesmo que esses tipos de atividades tenham tido uma queda durante a pandemia de COVID-19. Pois sabe-se que o câncer é a segunda maior causa de morte no mundo, com 9,6 milhões de mortes em 2018, sendo que cerca de 70\% ocorreram em países de baixa e média renda. Algumas iniciativas são de extrema importância voltada a promover o cuidado de pacientes com câncer, incluindo a prevenção ao manejo terapêutico especializado (Astigueta-Pérez et al., 2020).

Pacientes oncológicos, em especial aqueles que estão em fase de tratamento, são mais suscetíveis à infecção, devido a algumas modalidades terapêuticas que acarretam no comprometimento do sistema imunológico (Emami et al., 2020). Ferreira, Lima e Oliveira (2020), descreveram em seu estudo que há chance duas vezes maior na suscetibilidade da infecção por pacientes com câncer quando comparado com à população geral.

Um dos principais fatores que preocupam quando o assunto é pacientes com câncer é a incapacidade de receber os serviços médicos necessários. De acordo com o Relatório da Missão Conjunta OMS-China sobre COVID-19, os pacientes com câncer tiveram um risco estimado de 2 vezes maior de COVID-19 do que a população em geral. Pacientes com comorbidades tiveram taxas muito mais altas: $13,2 \%$ para aqueles com doença cardiovascular, $9,2 \%$ para diabetes, $8,4 \%$ para hipertensão, 8,0\% para doença respiratória crônica e 7,6\% para câncer (Shankar et al., 2020)

De acordo com uma pesquisa realizada na china, onde participaram 1.590 pacientes com COVID-19, dos quais 18 pacientes tinham quadro de câncer, observou-se que esses pacientes tiveram um risco maior de desenvolver eventos graves, como no caso de admissão na unidade de terapia intensiva, uso de ventilação invasiva ou progredir para quadro de óbito, quando comparado aos pacientes sem câncer: 39\% versus 8\%, respectivamente (Liang et al., 2020).

Alcântara et al. (2020) apontaram que os pacientes com câncer, quando idoso, possuem outros fatores de risco, como no caso da hipertensão e diabetes que, somados ao câncer, torna a COVID-19 torna-se bem mais agressiva. Notase que os pacientes que foram submetidos a tratamentos mais invasivos, como cirurgia e quimioterapia, apresentam um risco maior de evoluírem com eventos clínicos mais graves.

Foi observado por Zhang et al. (2020), que teve um total de 28 pacientes com câncer infectados com COVID-19, que $35,7 \%$ dos pacientes oncológicos tiveram quadro de estadiamento clínico IV do tumor e 21,4\% realizaram terapia antitumoral até 14 dias anteriores ao diagnóstico de COVID-19. Além disso, pode-se observar um risco elevado no desenvolvimento de infecção mais grave em pacientes em terapia antitumoral $(\mathrm{HR}=4,079 ; \mathrm{IC} 95 \%$ 1,086-15,322, $\mathrm{p}=0,037)$.

Xia et al. (2020), relataram que os pacientes com câncer estavam sujeitos a eventos graves do COVID-19. Isso como devido a inflamação avassaladora e a lesão pulmonar associada a citocinas podem ser importantes para instigar esses eventos graves em pacientes com COVID-19. Porém, nota-se que o desenvolvimento de câncer geralmente está associado a um estado imunológico fragilizado. Caracterizado pela indução suprimida de sinais de perigo pró-inflamatórios, maturação de células dendríticas prejudicada e populações de leucócitos imunossupressores funcionais elevadas. 
O mesmo estudo descreve que entre os tipos de cânceres, analisou nove artigos que possuíam informações clínicas e epidemiológicas de pacientes com câncer que foram infectados pelo novo coronavírus, totalizando 39 indivíduos. A partir disso, destacou-se o de pulmão, correspondendo a 51,3\% $(\mathrm{n}=20)$ dos pacientes, seguido do câncer de mama, com $10,3 \%$ $(n=4)$, e do câncer de cólon, com 7,7\% (n=3). Os demais tipos, quando agrupados, somaram os $28,7 \%(n=12)$ restantes (Figura 1).

Figura 1: Caracterização clínica e epidemiológica $(n=39)$.

\begin{tabular}{ccc}
\cline { 1 - 2 } Variável & N & $\%$ \\
\cline { 1 - 2 } Pulmão de câncer & 20 & 51,3 \\
Mama & 4 & 10,3 \\
Rim & 1 & 2,6 \\
Reto & 2 & 5,1 \\
Cólon & 3 & 7,7 \\
Colorretal & 1 & 2,6 \\
Pâncreas & 1 & 2,6 \\
Urotelial & 1 & 2,6 \\
Bexiga & 2 & 5,1 \\
Adrenal & 1 & 2,6 \\
Tireoide & 1 & 2,6 \\
Mieloma múltiplo & 1 & 2,6 \\
Linfoma & 1 & 2,6
\end{tabular}

Fonte: Adaptado de Alcântara et al., (2020).

Stevanato et al. (2021), tiveram como resultado de seu estudo o número total de óbitos de mulheres brasileiras por câncer de mama no período de 01 de janeiro a 15 de maio de 2020 foi de 5.522 , uma diferença de 336 óbitos em relação ao mesmo período do ano de 2019 que atingiu o total de 5.186 óbitos. Dos 336 óbitos, 69 (20,54\%) foram de câncer de mama associado a COVID-19.

Um estudo semelhante foi encontrado em uma pesquisa realizada no Reino Unido, onde mostrou que aproximadamente $6 \%$ no número de mortes por câncer de mama no período da pandemia, devido a consequência das medidas nacionais adotadas no período da pandemia do COVID-19, que reduziu a procura por cuidados de saúde, acesso e disponibilidade de serviços de diagnóstico, porém, os autores não associam as duas doenças. Além disso, um estudo realizado nos Estados Unidos, Canadá e Espanha descreveu que houve associação entre câncer de mama e COVID-19 com outros fatores de riscos gerais (Kuderer et al., 2020; Maringe,et al., 2020).

\section{Considerações Finais}

As pessoas com idade avançada e aquelas com doenças crônicas, como diabetes, doenças cardiovasculares e respiratórias, e com imunidade baixa, possuem um risco maior de terem complicações graves se forem contaminadas pelo coronavírus. Da mesma forma, pessoas com câncer que estejam em tratamentos de quimioterapia, radioterapia, que tenham 
feito cirurgia há menos de um mês ou que façam uso de medicamentos imunossupressores fazem parte do grupo de risco, tendo em vista que pacientes oncológicos são efetivamente mais vulneráveis a desenvolver a COVID-19 em sua foram mais severa.

O câncer, por si só, pode ser um estado que imunocompromete o paciente, e muitos tratamentos contra o câncer podem comprometer ainda mais o sistema imunológico. Por conta disso, pacientes com câncer com febre ou achados respiratórios inferiores, por exemplo, tosse, dispneia e hipóxia, estão entre as prioridades para o teste COVID-19, assim como os pacientes expostos a alguém testado positivamente para o COVID-19.

No entanto, necessita-se manter foco sobre a promoção da qualidade de vida e de morte desses pacientes e de seus familiares, pautando-se sobre a comunicação e o trabalho em equipe multiprofissional. Muitos esforços estão sendo empreendidos para melhor entender as particularidades dos pacientes com câncer infectados pelo novo coronavírus.

\section{Referências}

Al-Quteimat, OM, \& Amer, AM (2020). O impacto da pandemia COVID-19 em pacientes com câncer. Jornal americano de oncologia clínica .

Alcântara, R. C., Junior, L. C. F. S., Arnozo, G. M., de Oliveira, T. F., Santana, F. M. S., da Silva Filho, E. R., ... \& de Souza, C. D. F. (2020). Covid-19 em Pacientes oncológicos: uma Revisão do perfil Clínico-Epidemiológico. Revista Brasileira de Cancerologia, 66(TemaAtual).

Astigueta-Pérez, J., Abad-Licham, M., Chávez-Chirinos, C., Beraun-Milla, L., Lachos-Dávila, A., Diaz-Pérez, E., ... \& Mezones-Holguín, E. (2020). Cancer disease progression and death during the COVID-19 pandemic: a multidisciplinary analysis for the Peruvian setting. ecancermedicalscience, 14.

Emami, A., Javanmardi, F., Pirbonyeh, N., \& Akbari, A. (2020). Prevalence of underlying diseases in hospitalized patients with COVID-19: a systematic review and meta-analysis. Archives of academic emergency medicine, 8(1).

Ferreira, J. D., de Lima, F. C. D. S., Oliveira, J. F. P., de Camargo Cancela, M., \& de Oliveira Santos, M. (2020). Covid-19 e câncer: atualização de aspectos epidemiológicos. Revista Brasileira de Cancerologia, 66(TemaAtual).

Kuderer, N., Choueiri, T. K., Shah, D. P., Shyr, Y., Rubistein, S. M., et al. (2020). Clinical impact of COVID-19 on patient with cancer (CCC19): a cohort study. The Lancet, 395 (10241), 1907-1918.10.1016/S0140-6736(20)31187-9.

Liang, W., Guan, W., Chen, R., Wang, W., Li, J., \& Xu, K. (2020). Pacientes com câncer na infecção por SARS-CoV-2: uma análise nacional na China. Lancet Oncol, 21, 335-337.

Maringe, C., Spicer, J., Morris, M., Purushotham, A., Nolte, H., et al. (2020) The impact of the COVID-19 pandemic on cancer deaths due to delays in diagnosis in England, UK: a national, population-based, modelling study. The Lancet Oncology, 21(8), 1023-1034.10.1016 / S1470-2045 (20) 30388-0

Mendes, K. D. S., Silveira, R. C. D. C. P., \& Galvão, C. M. (2008). Revisão integrativa: método de pesquisa para a incorporação de evidências na saúde e na enfermagem. Texto \& contexto-enfermagem, 17, 758-764.

Pompeo, D. A., Rossi, L. A., \& Galvão, C. M. (2009). Revisão integrativa: etapa inicial do processo de validação de diagnóstico de enfermagem. Acta paulista de enfermagem, 22, 434-438.

Sampaio, S. G. D. S. M., Dias, A. M., \& de Freitas, R. (2020). Orientações do serviço médico de uma unidade de referência em cuidados paliativos oncológicos frente à pandemia de Covid-19. Revista Brasileira de Cancerologia, 66(TemaAtual).

Santos, A. M. R., \& Corrêa, F. M. (2020). Recomendações para detecção precoce de câncer durante a pandemia de covid-19 em 2021. Revista de APS, 23(1).

Shankar, A., Saini, D., Roy, S., Jarrahi, AM, Chakraborty, A., Bharati, SJ, \& Taghizadeh-Hesary, F. (2020). Desafios na prestação de cuidados com o câncer em meio ao surto da doença coronavírus 19 (COVID-19): precauções específicas para pacientes com câncer e prestadores de cuidados com o câncer para prevenir a disseminação. Jornal da Ásia-Pacífico de prevenção do câncer: APJCP , 21 (3), 569.

Silva, M. F., Silva, D. S. M. D., Bacurau, A. G. D. M., Francisco, P. M. S. B., Assumpção, D. D., Neri, A. L., \& Borim, F. S. A. (2021). Ageismo contra idosos no contexto da pandemia da covid-19: uma revisão integrativa. Revista de Saúde Pública, 55, 4.

Soares, K. H. D., da Silva Oliveira, L., da Silva, R. K. F., de Assis Silva, D. C., do Nascimento Farias, A. C., Monteiro, E. M. L. M., \& Compagnon, M. C. (2021). Medidas de prevenção e controle da covid-19: revisão integrativa. Revista Eletrônica Acervo Saúde, 13(2), e6071-e6071.

Stevanato, K. P., de Carvalho Dutra, A., dos Santos, L., Rosckovisk, I., Ribeiro, H. F., de Barros Carvalho, M. D., ... \& Pelloso, S. M. (2021). Perfil epidemiológico das mortes por câncer de mama e covid-19. Research, Society and Development, 10(8), e27210817269-e27210817269.

Xia, Y., Jin, R., Zhao, J., Li, W., \& Shen, H. (2020). Risk of COVID-19 for patients with cancer. The Lancet Oncology, 21(4), e180.

Zhang, L., Zhu, F., Xie, L., Wang, C., Wang, J., Chen, R., ... \& Zhou, M. (2020). Clinical characteristics of COVID-19-infected cancer patients: a retrospective case study in three hospitals within Wuhan, China. Annals of oncology, 31(7), 894-901.

Zuil, D. M., Fontoura, V. M., Santos, F. S., Neto, M. S., Pascoal, L. M., Martins, M. C. N. S. E., ... \& Graepp-Fontoura, I. (2021). Esquemas terapêuticos para combate da Covid-19: revisão sistemática. Research, Society and Development, 10(1), e21310111533-e21310111533. 